\title{
Pattern of Peripheral Neuropathy and B12 Assay among Type 2 Diabetic Saudi Patients
}

\author{
Ahmed Abdelbadie Mohammed ${ }^{* 1}$, Ahmed Abdualrhman Aljohani ${ }^{2}$, Jamil Ramadan Aljehany ${ }^{3}$, \\ Bothaina Omar Mara ${ }^{4}$, Abdullah Mohammad A Alsadiq ${ }^{5}$, Mohammed Abdualrhman Aljohani ${ }^{6}$, \\ Bothaina Omar Mara ${ }^{7}$
}

${ }^{1}$ Department Pathology Institute Taibah University; ahmedbadie60@yahoo.com

${ }^{2}$ Department, Medical student Institute Taibah University, Saudi Arabia; medicalahmed2050@ gmail.com

${ }^{3}$ Department, Supervisor of the Medical Conferences Unit, College of Medicine Institute, Taibah University,

Saudi Arabia; jrjehany@taibahu.edu.sa

${ }^{4}$ Department, General Surgery Institute, Taibah University, Saudi Arabia; bmaree@ taibahu.edu.sa

${ }^{5}$ Department, Medical Student Institute, Taibahu University, Kingdome of Saudia Arabia; ama91sa@gmail.com

${ }^{6}$ Department, Medical Student Institute Alrayan University, Saudi Arabia; maljohani386@gmail.com

${ }^{7}$ Department, General Surgery Institute, Taibah University, Saudi Arabia; bmaree@ taibahu.edu.sa

Corresponding author: Ahmed Abdelbadie Mohammed; ahmedbadie60@yahoo.com

Received: 03 October 2020;

Accepted: 23 October 2020;

Published: 01 November 2020

\begin{abstract}
Objectives: to determine whether the pattern of peripheral neuropathy among Saudi types 2 diabetics has association with B12 status and glycemic control. Method: A cross section hospital based study. The pattern of diabetic neuropathy was determined by nerve conductive velocity (NCV) test, level of vitamin b12 was assayed among the study population and the glycemic control was determined according to Hbalc level. Results: A total of thirty three patients were enrolled in these study twenty one females and twelve males. The age ranged between 79 and 34 with the mean (SD) of 57 of these $17(51.5 \%)$ used oral hypoglycemic agents and $16(48.5 \%)$ were using insulin. HbAc1 more than 7 was found in $28(84.8 \%)$ of the patients reflecting poor control. The nerve conduction study testing revealed that sensory axonal demyelination $6(18.2 \%)$, bilateral neuropathy $8(24.2 \%)$, Right Carpal tunnel syndrome 5(15.2\%), Left Carpal tunnel syndrome 0 (00\%), mild axonal neuropathy $4(12.1 \%)$ and $10(30.3 \%)$ were found to have normal nerve conduction study test. The level of vitamin B12 was found $2(6 \%)$ was deficiency $<180 \mathrm{pg} / \mathrm{ml}, 12(36.3 \%)$ possible deficiency $212 \mathrm{pg} / \mathrm{ml}-350 \mathrm{pg} / \mathrm{ml}$ and 19(57.5\%) was $>400 \mathrm{pg} / \mathrm{ml}$. Conclusion: It could be concluded from this study that there is no association between pattern of peripheral neuropathy and B12 level in type II diabetics. Similarly no relation exists between $\mathrm{Hb}$ Ac1 level and pattern of peripheral neuropathy.
\end{abstract}

Keywords: (type 2 diabetes, peripheral neuropathy, nerve conduction velocity test, B12)

\section{Introduction}

Saudi Arabia has one of the highest rates of diabetes worldwide, with an estimated number of 2,065,300 people diagnosed with the disease by the end of 2010 , which constitutes around $16.8 \%$ of the population. Nevertheless, the exact number swings taking into account figures emerged from different studies. Type 2 diabetic patients were around $85.2 \%$ of all diabetics. $62.7 \%$ of them had symptomatic diabetic peripheral neuropathy ${ }^{[1]}$.

Diabetic peripheral neuropathy (DPN) is a common complication of diabetes that is associated with axonal atrophy, demyelination, blunted regenerative potential, and loss of peripheral nerve fibers. The development and progression of DPN is due in large to hyperglycemia but is also affected by insulin deficiency and dyslipidemia. Although numerous biochemical mechanisms contribute to DPN, increased oxidative/nitrosative stress and mitochondrial dysfunction seem intimately associated with nerve dysfunction and diminished regenerative capacity ${ }^{[2]}$. A positive correlation was shown by linear regression charts between the grades of nerve conduction defects in asymptomatic diabetic neuropathy patients and duration of diabetes, age, BMI, and HbA1C ${ }^{[3]}$. Deficiency of vitamin B6 (PLP, pyridoxine, pyridoxal) and vitamin B1 (thiamine) were prevalent in type 2 diabetes. The primary stage of nephropathy was associated with more pronounced alterations in vitamin B6 metabolism and stronger indications of endothelial dysfunction and inflammation ${ }^{[4]}$.

In fact, vitamin B12 deficiency was documented in association with peripheral neuropathy, visual failure with optic 
atrophy without retinopathy. Diabetic neuropathy was found in relation to combined degeneration of the cord ${ }^{[4,5]}$.

Good glycemic control was associated with reduced neuropathy in both type 1 and type 2 diabetics ${ }^{[5]}$.

Vitamin B12 (cobalamin) is necessary for hematopoiesis and normal neuronal function. In humans, it is obtained only from animal proteins and requires intrinsic factor (IF) for its absorption. The body used vitamin B12 stores very economically, reabsorbing vitamin B12 from the ileum and returning it to the liver; very little is excreted. The amount excreted ranges between 180-914 ng/ ${ }^{[6]}$. Vitamin B12 deficiency was defined as serum concentration of $<200 \mathrm{pg} / \mathrm{dl}$, borderline deficiency as $200-300 \mathrm{pg} / \mathrm{dl}$ and $>300$ $\mathrm{pg} / \mathrm{dl}$ as normal. It was found that the level of vitamin B12 decreased by the use of metformin but it did not affect the incidence of neuropathy among diabetics ${ }^{[7]}$.

Vitamin B 12 level has been found to correlate with many diseases like multiple sclerosis and thyroid hormone deficiency. In a disease like peripheral neuropathy in which the etiological factors are complex, it's difficult to attribute the disease to a single culprit. Evidence pointed to the role of vitamins B groups in the process. it is questionable whether that normalization of B12 level may brought improvement. So far the level of vitamin B12 among Saudi subjects with type 2 diabetes is not investigated and the exact role of vitamin B12 in neuropathy among diabetic's need to be determined.

In the present study, was assessed severity of diabetic neuropathy by nerve conductive velocity (NCV) test, level of vitamin b12 was assayed among the study population. The glycemic control was determined from Hba1c level.

\section{Materials and methods}

A cross section hospital based study was conducted in King Fahad Hospital Neurology Department at Almadinah ALmunararah during period from May to August 2018.

Thirty three type 2 diabetic patients males and females were consented to participated in the study. Demographic characteristics were gathered using questionnaire.

Nerve conduction measurement: nerve conduction velocity (NCS) test

Nerve Conduction Studies were done at room temperature of $23 \pm$ $2{ }^{\circ} \mathrm{C}$. For all studies the machine used was Keypoint work station (Medtronic, France). Simplified nerve conduction studies (NCS) protocol was followed ${ }^{[11,12]}$.

Assay of vitamin B12: Assay of vitamin B 12 was done at King Fahad Hospital laboratory (using the Roche - Cobas e 601-USA). The assay was quantitative, values were reported in the range between less than 180 to more than $400 \mathrm{ng} / \mathrm{ml}$. Precision was determined using Elecsys reagents, pooled human sera and controls in a protocol (EP5A2) of the CLSI (Clinical and Laboratory Standards Institute.

Table 1: Shows the characteristics of the study population $(n=33)$

\begin{tabular}{|c|c|c|c|}
\hline \multicolumn{2}{|l|}{ Variable } & \multirow{2}{*}{$\begin{array}{l}\text { Frequency } \\
\mathbf{N}=\mathbf{3 1} \\
12\end{array}$} & \multirow{2}{*}{$\begin{array}{l}\text { Percent \% } \\
36.4 \%\end{array}$} \\
\hline Sex & Male & & \\
\hline sex & Female & 21 & $63.6 \%$ \\
\hline \multirow{2}{*}{ Hypoglycemic therapy } & Oral & 17 & $51.5 \%$ \\
\hline & Insulin & 16 & $48.5 \%$ \\
\hline \multirow{2}{*}{ Previous operation } & Yes & 8 & $24.2 \%$ \\
\hline & No & 25 & $75.8 \%$ \\
\hline \multirow{2}{*}{ HBa1c } & $>7$ & 28 & $84.8 \%$ \\
\hline & $<$ or $=7$ & 5 & $15.2 \%$ \\
\hline
\end{tabular}

\section{Statistics}

Data were entered in computer using SPSS for windows version 19 for analysis. Frequencies and means were determined using $\mathrm{T}$ test. A p value of less than 0.05 was significant. Correlations were determined using Spearman's correlation coefficient to compare between ranks, the association was strong at $1+$ (for positive association) and 1- (for negative association).

\section{Ethics}

The study had ethical approval from the ethical committee at the faculty of medicine Taibah University.

\section{Results}

\section{General characteristics}

The thirty three patients enrolled in this study were twenty one females and twelve males. The age ranged between 79 and 34 with the mean(SD) of 57 of these $17(51.5 \%)$ used oral hypoglycemic agents and $16(48.5 \%)$ were using insulin. HbAc1 more than 7 was found in $28(84.8 \%)$ of the patients reflecting poor control. The nerve conduction study testing revealed that sensory axonal demyelination $6(18.2 \%)$, bilateral neuropathy 8 (24.2\%), Right Carpal tunnel syndrome 5(15.2\%), Left Carpal tunnel syndrome 0 (00\%), mild axonal neuropathy $4(12.1 \%)$ and $10(30.3 \%)$ were found to have normal nerve conduction study test. The level of vitamin B12 was found 2(6\%) was deficiency $<180 \mathrm{pg} / \mathrm{ml}$, 12(36.3\%) possible deficiency $212 \mathrm{pg} / \mathrm{ml}-350 \mathrm{pg} / \mathrm{ml}$ and $19(57.5 \%)$ was $>400 \mathrm{pg} / \mathrm{ml}$.(Table 1)

\section{Nerve conduction study test (NCST) and Hb Ac1}

The correlation between the ranks of NCST and HbAcl was done along the scale of Spearman correlation coefficient which ranges between $1+$ and 1- which indicates the strength of association between the variables. The results of spearman test were -0.199 , $0.04,-0.179,0.36$ and 0.089 . there was weak positive association between mild axonal neuropathy and $\mathrm{HbAcl}$ other parameters of the NCST showed insignificant correlation with the level of HBAC1.(Table 2)

\section{Nerve conduction study test and B12 assay}

The correlation between B12 assay and NCST using Spearman's correlation coefficient revealed a positive weak correlation between sensory axonal demyelination and B12 level that means it increases with increase in B12 level.

Also a negative weak correlation was found between right carpal tunnel syndrome and B12 assay (Spearman's - 0.346) that means carpal tunnel syndrome decreases with decrease in B12 level (Table 3). 


\begin{tabular}{|l|l|l|l|}
\hline \multirow{4}{*}{ Nerve conduction testing(NCS) } & Sensory Axonal Demyelinating & 6 & $18.2 \%$ \\
\cline { 2 - 4 } & Bilateral Neuropathy & 8 & $24.2 \%$ \\
\cline { 2 - 4 } & Right Carpal tunnel syndrome & 5 & $15.2 \%$ \\
\cline { 2 - 4 } & Left Carpal tunnel syndrome & 0 & $0.0 \%$ \\
\cline { 2 - 4 } & Mild Axonal Neuropathy & 4 & $12.1 \%$ \\
\cline { 2 - 4 } B12 assay & Normal & 2 & $30.3 \%$ \\
\hline & deficiency $<\mathbf{1 8 0} \mathbf{~ p g} / \mathbf{m l}$ & 12 & $6 \%$ \\
\cline { 2 - 4 } & possible deficiency $\mathbf{2 1 2} \mathbf{~ p g / m l - 3 5 0 ~} \mathbf{~ g g / m l}$ & 19 & $36.3 \%$ \\
\cline { 2 - 4 } & $\mathbf{> 4 0 0} \mathbf{~ p g / m l}$ & $57.5 \%$ \\
\hline
\end{tabular}

Table 2: Shows the correlation between HBA1C level and pattern of peripheral neuropathy determined by NCST.

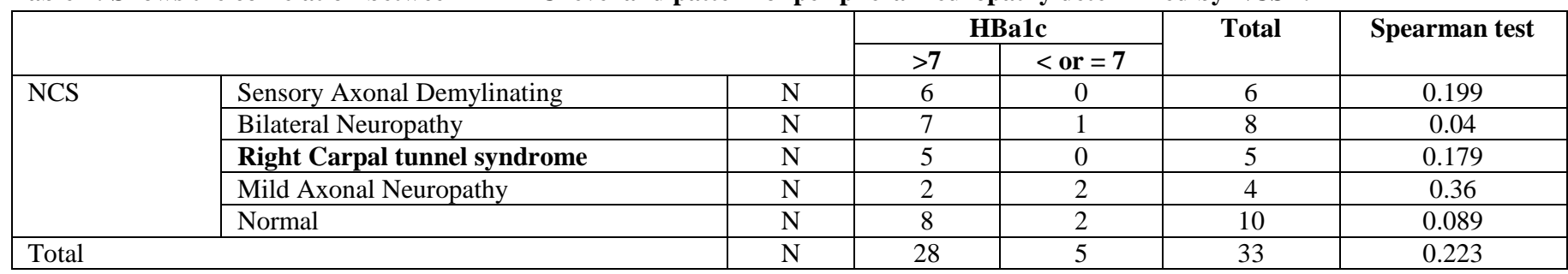

Table 3: Shows the correlation between B12 deficiency and pattern of neuropathy determined by NCST

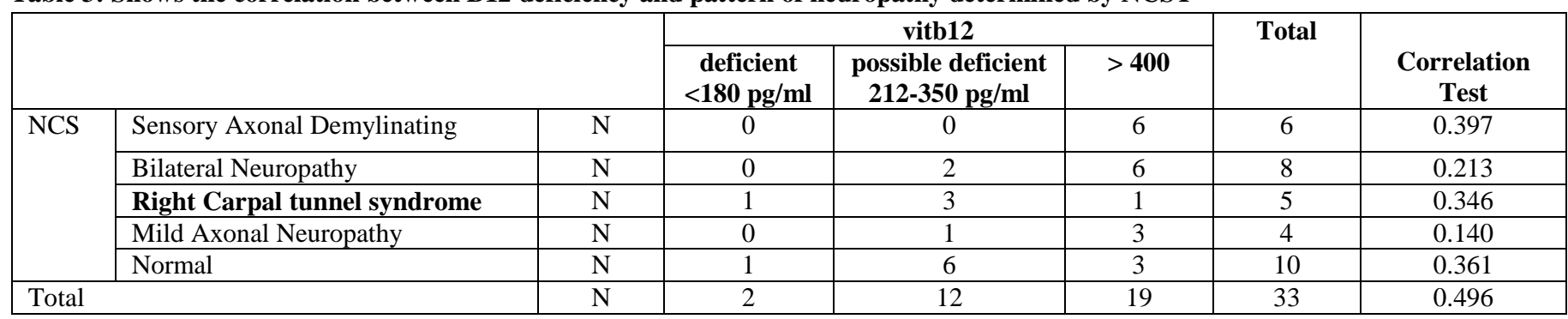

\section{Discussion}

Peripheral neuropathy is a common complication of DM, with impairment of quality of life and high morbidity. The determination of modifiable risk factors improves life quality. The population assessed in this study showed both bad control as indicated by $\mathrm{HbAc1}(>7$ in $84 \%$ ) and B12 deficiency (42\%).

Leihsear et al. found vitamin B12 deficiency in $17.1 \%$ of old adults without diabetes, they took lower cut off point(less than $260 \mathrm{pmol} / \mathrm{L}$ ) and measured MMA (methyl malonic acid) which is more sensitive parameter of B12 deficiency. They correlated B12 defiency with PN. Their finding were not in agreement with our findings in term of no association was found between PN and B12 defiency.

NCVT is sensitive indicator of demyelination and no assessment of sensory function was included in our study. The results are applicable to motor neuropathy. It is more this hospitalbased study found a considerable prevalence of PN among uncontrolled diabetic patients $(84.8 \%)$ In other hospital based study show (29.7\%) of type 2 diabetic patients complain of PN.

Mathew and co-workers found that 22\% DM type 2 patients have B12 deficiency ${ }^{[12]}$ and another one poor B12 (deficient B12 status and low serum B12) is associated with worse sensory and motor peripheral nerve function. Nerve function impairments may lead to physical function declines and disability in elderly ${ }^{[13]}$.

Our findings showed that $6.1 \%$ of peripheral neuropathy patients had B12 defiency and vitamin B12 has a statistically significant negative relationship with the severity and class of diabetic neuropathy

Previous studies have demonstrated that supplemental vitamin B12 improved somatic and autonomic symptoms of diabetic neuropathy ${ }^{[23]}$. Treating, B12 deficiency in those patients with neuropathy may lead to improved clinical outcomes.
This study did not address the previous use of B12 vitamin supplementation that would affected the values of B12 assay.it focused on the current severity of the disease versus the current B12 status.

Limitations of this study include the small sample size and non- inclusion of matched controls for each type of peripheral neuropathy. Sensory nerve function was not assessed.

\section{Conclusion}

It could be concluded from this study that there is no relation between pattern of peripheral neuropthay and B12 level in type II diabetics. Similarly no relation exists between $\mathrm{Hb}$ Ac1 level and pattern of peripheral neuropathy.

\section{Ethics approval and consent to participate}

Ethics approval and consent taken from Taibah University

\section{List of abbreviations}

Diabetic peripheral neuropathy (DPN)

Body mass index (BMI)

Intrinsic factor (IF)

Nerve conductive velocity (NCV)

Clinical and Laboratory Standards Institute.(CLSI)

MMA (methyl malonic acid)

\section{Data Availability}

A data availability statement is from files and data base at king Fahad Hospital at Madina 


\section{Conflicts of Interest}

There is no conflict of interest,

\section{Funding Statement}

None

\section{Authors' contributions}

Ahmed Abdelbadie Mohammed: Analysis and interpretation of date have been involved in drafting the manuscript or revising it critically for important intellectual content have given final approval of the version to be published agree to be accountable for all aspects of the work in ensuring that questions related to the accuracy or integrity of any part of the work are appropriately investigated and resolved.

\begin{abstract}
Ahmed Abdualrhman Aljohani: Have made substantial contributions to conception and design, or acquisition of data, or analysis and interpretation of date have been involved in drafting the manuscript or revising it critically for important intellectual content have given final approval of the version to be published agree to be accountable for all aspects of the work in ensuring that questions related to the accuracy or integrity of any part of the work are appropriately investigated and resolved.
\end{abstract}

Mohammed Abdualrhman Aljohani: Analysis and interpretation of date have been involved in drafting the manuscript or revising it critically for important intellectual content have given final approval of the version to be published agree to be accountable for all aspects of the work in ensuring that questions related to the accuracy or integrity of any part of the work are appropriately investigated and resolved.

Jamil Ramadan Aljehany: have given final approval of the version to be published agree to be accountable for all aspects of the work in ensuring that questions related to the accuracy or integrity of any part of the work are appropriately investigated and resolved.

Bothaina Omar Mara: have given final approval of the version to be published agree to be accountable for all aspects of the work in ensuring that questions related to the accuracy or integrity of any part of the work are appropriately investigated and resolved.

Abdullah Mohammad A Alsadiq: have given final approval of the version to be published agree to be accountable for all aspects of the work in ensuring that questions related to the accuracy or integrity of any part of the work are appropriately investigated and resolved.

\section{Acknowledgement}

Author would like to thank Doctor Mohammed Qrimle Neurology Consultant King Fahad hospital who did the assessment and NCST.

\section{References}

1. Diabetes Atlas (2012) Global and Regional Diabetes Statistics

2. Kevin L. Farmer, Chengyuan Li, and Rick T. Dobrowsky."Diabetic Peripheral Neuropathy: Should a Chaperone Accompany Our Therapeutic Approach? Journal 2012; 880-900.

3. Mariko Kobori, and co-worker. Four-year sequential nerve conduction changes since first visit in Japanese patients with early type 2 diabetes. sciencedirect. 2017; 369-376.

4. A.I. Adler, R.J. Stevens, S.E. Manley, R.W. Bilous, C.A. Cull, R.R. HolmanDevelopment and progression of nephropathy in type 2 diabetes: the United Kingdom Prospective Diabetes Study. Kidney 2003; pp. 225-232.

5. Callah an BC, Cheng HT, Stables C, Smith AL, Feldman EI. Diabetic neurop athy : clinical manif estations and current treatments. Lancet Neurol 2012; 11:521-534.

6. Babior BM: The megaloblastic anemias. In Hematology. Fifth edition. Edited by WJ Williams, E Beutler, MA Lichtman, et al. New York, McGraw-Hill Book Company, 1995; pp 471-490.

7. Sedar Olt,orhan Oznas. Investigation of the vitamin B12 deficiency with peripheral neuropathy in patients with type 2 diabetes mellitus treated using metformin. North Clin Istanb. 2017; 4(3): 233-236.

8. Al-Nozha MM, Arafah MR, Al-Mazrou YY, Al-Maatouq MA, Khan NB, Khalil MZ, Al- Khadra AH, AlMarzouki K, Abdullah MA, Al-Harthi SS, et al. Coronary artery disease in Saudi Arabia. Saudi Med J. 2004; 25(9): 1165-71.

9. Sample size calculation: Cross-sectional studies [Internet]. communitymedicine4asses. 2018 [cited 15 May 2018]. Available from: https://communitymedicine4asses.com/2014/05/11/sampl e-size-calculation-cross-sectional-studies/

10. Bosse MJ, McCarthy ML, Jones AL, et al The insensate foot following severe lower extremity trauma: an indication for amputation? J Bone Joint Surg Am 2005;87:2601-8

11. Perkins BA, Vera Bril. Diabetic neuropathy: a review emphasizing diagnostic methods. Clin Neurophysiol 2003; 114: 1167-75.

12. Matthew C. Pflipsen, Robert C. Oh, Aaron Saguil et al. The Prevalence of Vitamin B12 Deficiency in Patients with Type 2 Diabetes. J Am Board Fam Med. September-October 2009 vol. 22 no. 5 528-534

13. Kira Leishear, Robert M. Boudreau, Stephanie A. Studenski et al. The Relationship of Vitamin B12 and Sensory and Motor Peripheral Nerve Function in Older Adults. J Am Geriatr Soc. 2012 Jun; 60(6): 1057-1063. 